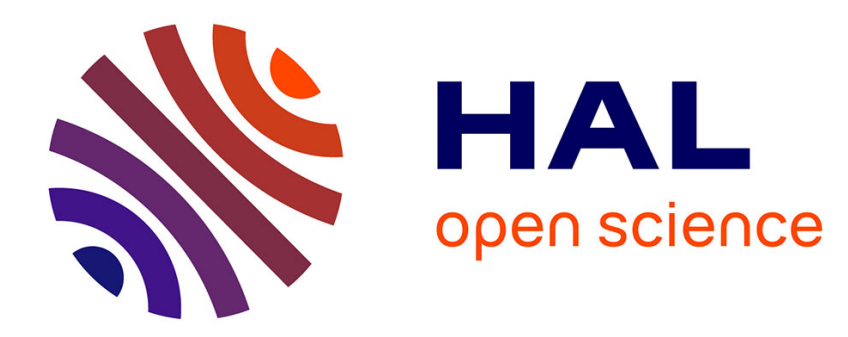

\title{
MODULATION SPECTROSCOPY IN THE VACUUM ULTRAVIOLET
}

\author{
D. Lynch
}

\section{To cite this version:}

D. Lynch. MODULATION SPECTROSCOPY IN THE VACUUM ULTRAVIOLET. Journal de Physique Colloques, 1978, 39 (C4), pp.C4-125-C4-133. 10.1051/jphyscol:1978416 . jpa-00217450

\section{HAL Id: jpa-00217450 https://hal.science/jpa-00217450}

Submitted on 1 Jan 1978

HAL is a multi-disciplinary open access archive for the deposit and dissemination of scientific research documents, whether they are published or not. The documents may come from teaching and research institutions in France or abroad, or from public or private research centers.
L'archive ouverte pluridisciplinaire HAL, est destinée au dépôt et à la diffusion de documents scientifiques de niveau recherche, publiés ou non, émanant des établissements d'enseignement et de recherche français ou étrangers, des laboratoires publics ou privés. 


\title{
MODULATION SPECTROSCOPY IN THE VACUUM ULTRAVIOLET
}

\author{
D. W. LYNCH \\ Ames Laboratory-ERDA and Department of Physics, \\ Iowa State University, Ames, Iowa 50011, U.S.A.
}

\begin{abstract}
Résumé. - Cette revue est consacrée à la spectroscopie UVL utilisant les techniques de modulation. La thermomodulation a été employée pour l'étude des métaux et la modulation de composition pour les alliages. Les semiconducteurs ont été étudiés par thermomodulation et électromodulation; quand les niveaux de cœur sont utilisés comme états initiaux, cette dernière méthode apporte de nombreuses informations sur les bandes de conduction. Les isolants ont été étudiés par électro et thermomodulation (la modulation en longueur d'onde et la dérivation par rapport à l'énergie ne seront que brièvement évoquées). Dans tous les cas, des matériaux simples ont été étudiés, les avantages et inconvénients des différentes méthodes étant soulignés.
\end{abstract}

\begin{abstract}
A review of modulation spectroscopy in the vacuum ultraviolet is presented. Metals have been studied by thermomodulation spectroscopy and alloys by composition modulation Semiconductors have been studied by thermomodulation and by electromodulation, the latter revealing a great deal about the conduction bands when core levels are used as initial states. Insulators have been studied by electro and thermo-modulation. (Wavelength modulation and energy derivative modulation are not discussed extensively.) In all cases simple materials have been studied and the advantages and disadvantages of the methods delineated.
\end{abstract}

1. Introduction. - Modulation spectroscopy [1] is a technique whereby a solid sample is subjected to a periodic perturbation which alters its electronic structure, resulting in a small, but detectable, periodic change in the optical properties of the sample. The resultant modulation or differential spectrum has two advantages over conventional spectra : it usually resembles a derivative of the ordinary spectrum, making small structures enhanced, and it contains information on how the electronic structure of the sample is altered by the perturbation, information which aids in the elucidation of the electronic structure itself. We outline briefly a typical experiment and the data treatment, then discuss applications in the vacuum ultraviolet.

2. Methods. - An incident beam of intensity $I(\lambda)$ is reflected (or transmitted) by the sample. A perturbation, e.g., $F_{0} \cos \omega t$, is applied to the sample and $\Delta R(\lambda)$, a small change in the reflectance, $R(\lambda)$, may result. It will occur at the frequency $\omega$ or a harmonic, and may be shifted in phase by $\gamma$ with respect to the perturbation. The reflected beam,

$$
I(\lambda)[R(\lambda)+\Delta R(\lambda) \cos (\omega t+\gamma)],
$$

is detected and the d.c. and a.c. components separated. The latter, typically $10^{-3}$ to $10^{-5}$ of the former, is rectified synchronously with the perturbation. The ratio of the a.c. to d.c. signals is $\Delta R / R$, independent of the wavelength dependence of the incident flux and of the response of the detector.

Continuing with reflectance as our example, for only thin films transmit well into the vacuum ultraviolet, we begin with the Fresnel coefficients which give the ratio of reflected to incident electric fields :

$$
\begin{aligned}
& \widetilde{r}_{\mathrm{s}}=\left(\tilde{n}_{\mathrm{a}_{\perp}}-\widetilde{n}_{\perp}\right) /\left(\tilde{n}_{\mathrm{a}_{\perp}}+\tilde{n}_{\perp}\right) \\
& \widetilde{r}_{\mathrm{p}}=\left(\widetilde{\varepsilon} \tilde{n}_{\mathrm{a}_{\perp}}-\widetilde{\varepsilon}_{\mathrm{a}} \tilde{n}_{\perp}\right) /\left(\widetilde{\varepsilon} \tilde{\mathrm{a}}_{\mathrm{a}_{\perp}}+\widetilde{\varepsilon}_{\mathrm{a}} \tilde{n}_{\perp}\right),
\end{aligned}
$$

with $\varphi$ the angle of incidence from the ambient medium a and

$$
\begin{aligned}
\tilde{n}_{\mathrm{a}_{\perp}} & =\widetilde{n}_{\mathrm{a}} \cos \varphi \\
\tilde{n}_{\perp} & =\left(\widetilde{\varepsilon}-\widetilde{\varepsilon}_{\mathrm{a}} \sin ^{2} \varphi\right)^{1 / 2} .
\end{aligned}
$$

The complex dielectric functions and refractive indices $\widetilde{\varepsilon_{\mathrm{a}}}=\widetilde{N}_{\mathrm{a}}^{2}$ and $\widetilde{\varepsilon}=\tilde{N}^{2}=\varepsilon_{1}+i \varepsilon_{2}$ characterize the ambient and sample, here taken to be a cubic crystal or isotropic sample. The measured reflectances are $R_{\mathrm{s}}=\left|\widetilde{r_{\mathrm{s}}}\right|^{2}$ and $R_{\mathrm{p}}=\left|\widetilde{r_{\mathrm{p}}}\right|^{2}$. The applied perturbation causes $\widetilde{\varepsilon}$ to change to $\widetilde{\varepsilon}+\Delta \widetilde{\varepsilon}$. The change will be a tensor for some perturbations, even for a scalar $\tilde{\varepsilon}$. Straightforward differentiation of the expressions above leads to

$$
\frac{\Delta R}{R}+2 i \Delta \theta=[(\alpha-i \beta) \hat{\mathbf{e}} \cdot \Delta \widetilde{\varepsilon} \cdot \hat{\mathbf{e}}]
$$


where $\hat{\mathbf{e}}$ is the unit polarization vector of the incident beam and $\alpha-i \beta$ are the Seraphin coefficients $[2,3]$ :

$$
\begin{aligned}
& (\alpha-i \beta)_{\mathrm{s}}=\left(2 \widetilde{n}_{\mathrm{a}} \cos \varphi\right) / \tilde{n}_{\perp}\left(\widetilde{\varepsilon}-\widetilde{\varepsilon}_{\mathrm{a}}\right) \\
& (\alpha-i \beta)_{\mathrm{p}}=\frac{2 \tilde{n}_{\mathrm{a}}\left(\tilde{\varepsilon}-2 \widetilde{\varepsilon}_{\mathrm{a}} \sin ^{2} \varphi\right) \cos \varphi}{\tilde{n}_{\perp}\left(\widetilde{\varepsilon}^{2} \cos ^{2} \varphi-\widetilde{\varepsilon} \widetilde{\varepsilon}_{\mathrm{a}}+\widetilde{\varepsilon}_{\mathrm{a}}^{2} \sin ^{2} \varphi\right)} .
\end{aligned}
$$

All of these have been generalized to the case in which there is an intermediate layer of another material between the sample and the ambient, a layer representing an oxide, or a film that has been deliberately applied to the sample $[3,4]$.

A measured $\Delta R / R$ spectrum does not allow the recovery of a spectrum of $\Delta \widetilde{\varepsilon}$, for the real and imaginary parts of the latter are mixed in the former. It is possible to make a second measurement, e.g., of modulated transmission through a thin film, or of modulated reflectance at another angle of incidence, but the most widely use method is to Kramers-Kronig analyze the measured modulated reflectance spectrum [5]. Since

$$
\tilde{r}=r \mathrm{e}^{i \theta}, \quad \ln \tilde{r}=\ln r+i \theta .
$$

$\theta$ can be obtained from $\ln r=\frac{1}{2} \ln R$ by a Kramers-Kronig integral. With a perturbation applied, $\tilde{r} \rightarrow \tilde{r}+\Delta \tilde{r}$, with $r \rightarrow r+\Delta r$ and $\theta \rightarrow \theta+\Delta \theta$. Finally

$$
\Delta \theta(E)=\frac{E}{\pi} P \int_{0}^{\infty}\left(\frac{\Delta R\left(E^{\prime}\right)}{R\left(E^{\prime}\right)}-\frac{\Delta R(E)}{R\left(E^{\prime}\right)}\right) \frac{\mathrm{d} E^{\prime}}{E^{\prime 2}-E^{2}} .
$$

With the measured $\Delta R / R$ and calculated $\Delta \theta$ spectra, one can obtain the modulated dielectric function spectrum via equation (3). However, this requires the spectrum of $\tilde{\varepsilon}$ for the substrate itself, as well as for any intervening layer of a third material. Thus modulation spectroscopy is a second generation measurement, requiring previous or simultaneous measurements leading to the dielectric function of the sample. It is also useful to compute $\Delta[\operatorname{Im}(-1 / \tilde{\varepsilon})]$, the differential electron energy loss function, from $\Delta \widetilde{\varepsilon}$, for the former represents the effect of the perturbation on longitudinal excitations, and the latter, its effect on transverse excitations. $\Delta \widetilde{\varepsilon}$ or $\Delta(-1 / \tilde{\varepsilon})$ can be calculated from microscopic models for the solid, and are the spectra one should interpret. (One rarely, if at all, should interpret $\Delta R / R$, for the positions, shapes, and numbers of peaks in it and in the $\Delta \varepsilon_{1}$ and $\Delta \varepsilon_{2}$ spectra may be different.)

Because $\Delta R$ is small, the a.c. signal, $I \Delta R$, is small, and care must be taken to eliminate all spurious contributions to it, e.g. those arising from sample vibration or noise in the electronics. If this is done, the remaining source of noise is shot noise in the incident beam and from the photocathode. If $N$ photons are counted to determine a point on the $\Delta R / R$ spectrum, the signal-to-noise ratio will be of the order of $N^{1 / 2}$, with a factor of the order of unity accounting for the modulation waveform. If $R$ and $\Delta R$ are fixed, the signal-to-noise ratio can be increased only by increasing the intensity of the incident beam. Hence intense, stable (to avoid additional noise), continuous light sources are necessary.

In fact, $\Delta R / R$ is not fixed, because one can vary the angle of incidence and the polarization of the measuring light. The effect of so doing has been discussed by Aspnes [3, 4]. For shot-noise limited detection, the signal-to-noise ratio is proportional to the sensitivity function, $S=R^{1 / 2}|\alpha-i \beta|$, which depends on the angle of incidence, the polarization, and the dielectric functions of the sample and ambient. Given a wavelength, this can be plotted as a function of angle of incidence, and a maximum sought. For absorbing media in the visible, there is not much angle dependence, except for a fall-off to zero at grazing incidence, where there can be no modulation of the constant unit reflectance. In the vacuum ultraviolet $|\widetilde{\varepsilon}|$ approaches unity and $S$ exhibits a maximum (Fig. 1) which may be several times larger

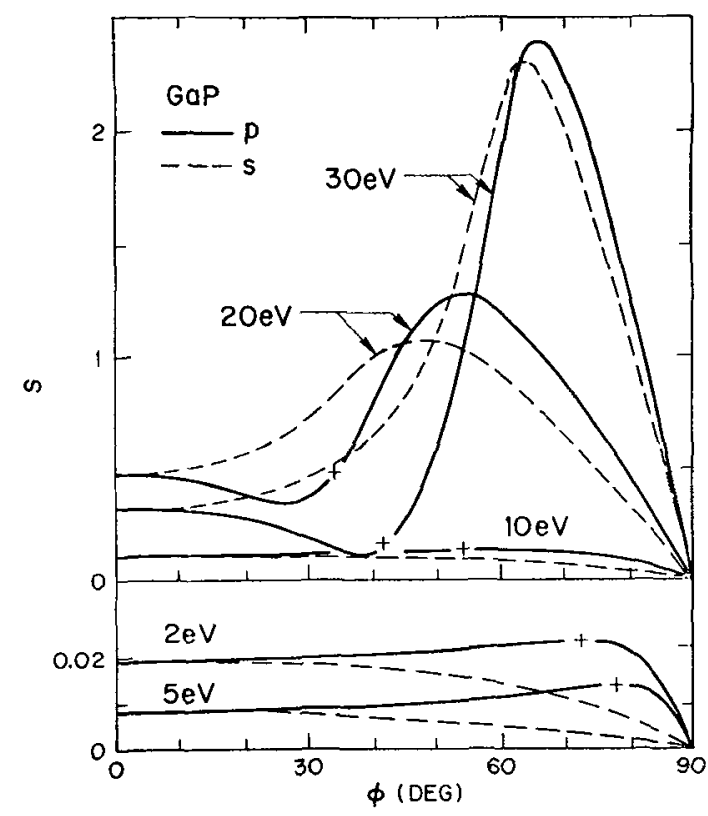

Frg. 1. - Dependence of the sensitivity function $S$ upon polarization and angle of incidence for GaP at five photon energies. The crosses indicate the angles at which the p-polarized reflectance is a minimum. (From Ref. [4].)

than the value at normal incidence. It should be noted that the maxima occur at angles considerably larger than the angles at which $R_{\mathrm{p}}$ has a minimum. This minimum increases $\Delta R / R$, but the reflected beam is then weak and the shot noise is therefore high. Moreover, there is no reflectance minimum for s-polarization but there is an angle at which $S$ can have an appreciable maximum. For further details and other examples, consult reference [4].

In general, the larger contributions to a modulated dielectric function come from groups of states which 
all respond in the same way to the applied perturbation. These usually are found at and near critical points in the band structure. The critical points lead to characteristic structures in $\Delta \varepsilon_{1}$ and $\Delta \varepsilon_{2}$. If the perturbation shifts the energy of the interband critical point, the modulated $\Delta \tilde{\varepsilon}$ spectra have characteristic shapes, those of the energy derivatives of the critical point contributions to $\Delta \widetilde{\varepsilon}$. These well-known shapes are sought in the measured spectra. If the perturbation broadens the transitions at critical points (or excitons). The characteristic signatures of these appear as first (or second) derivatives of their contributions to $\Delta \widetilde{\varepsilon}$. (Electroreflectance can give third derivative spectra.) Both first and second derivatives may occur together, or neither may occur if the critical point states are not affected by the perturbation. Other types of interband transitions may contribute to the $\Delta \widetilde{\varepsilon}$ spectrum. In these cases one must calculate or estimate the effect on these transitions to obtain the lineshape in $\Delta \varepsilon$ and the expected strength. The sharpness of the structures in $\Delta \varepsilon$, the occurrence of some characteristic lineshapes, and the fact that some expected features are very large or missing lead to the great utility of modulation spectroscopy in understanding the band structure of solids. We comment below on some of the types of modulation that can be applied. Some of their advantages and limitations are summarized in table $\mathrm{I}$.

2.1 Temperature modulation. - [6] may be applied to any sample. Such measurements are easy to carry out by modulating with direct or indirect resistive heating, by infrared heating, or by electron beam heating. Long thermal time constants require modulating at low frequencies $(\$ 15 \mathrm{~Hz})$ where $1 / f$ noise may dominate shot noise. Interpretation of the scalar $\Delta \tilde{\varepsilon}$ spectra is more complicated than for other perturbations because the temperature change has many effects. In semiconductors and insulators the dominant mechanism is usually the modulation of band gap energies, while in metals, thermal broadening and the broadening of the Fermi function edge commonly contribute as well [7].

2.2 Stress modulation. - [8-10] usually means uniaxial stress, and it may be applied, in principle, to any sample. By the use of single crystals and polarized radiation, considerable symmetry information may be obtained about the interband transitions. Because elastic strain limits on single crystals are so low, signals are small. It is common to apply a static stress and use another modulation technique to obtain the spectra at different static stresses. Metals exhibit little critical point structure, and their corresponding stress modulation spectra are very small and broad, only $\mathrm{Cu}$ [11] and $\mathrm{Au}$ [12], with relatively sharp structures in their $\widetilde{\varepsilon}$ spectra having yielded stress modulation spectra to date. No vacuum UV stress modulation spectroscopy has yet been carried out.

2.3 EleCtRIC FIELD MOdULATION. - [13-20] may be applied to non-metals. For insulators the technique is uncertain, for there can be large stresses intro-

TABLE I

\section{Modulation spectroscopies}

\begin{tabular}{|c|c|c|c|}
\hline Perturbation & $\Delta \tilde{\varepsilon}$ & Typical spectra & Advantages \\
\hline - & - & - & - \\
\hline Temperature & Scalar & $\begin{array}{l}1 \text { st and } 2 n d \text { deri- } \\
\text { vatives }\end{array}$ & Universal \\
\hline Hydrostatic pressure & Scalar & 1 st derivative & $\begin{array}{l}\text { Gives deformation } \\
\text { potentials }\end{array}$ \\
\hline Composition & Scalar & $\sim 1 s t$ derivative & \\
\hline Uniaxial stress & Tensor & 1 st derivative & $\begin{array}{l}\text { Gives symmetry in- } \\
\text { formation, defor- } \\
\text { mation potentials }\end{array}$ \\
\hline Electric field & Tensor & $3 r d$ derivative & High resolution \\
\hline Magnetic field & Tensor & $\begin{array}{l}1 \text { st derivative in } \\
\text { paramagnetics, } \\
\text { complicated in } \\
\text { ferromagnetics }\end{array}$ & \\
\hline Wavelength & Scalar & 1st derivative & Universal \\
\hline Energy derivative & Scalar & $\begin{array}{l}1 s t, 2 n d, 3 r d \text { deri- } \\
\text { vatives }\end{array}$ & Universal \\
\hline
\end{tabular}
Higher noise, interpretation may be complicated
Pressure medium required
Surfaces may limit sensiti- vity
Difficult to do except on some semiconductors
Limited types of samples Small signals except for ferro- magnetics

$\left\{\begin{array}{l}\text { Give no response to pertur- } \\ \text { bation }\end{array}\right.$ 
duced by the electric field, leading to a mixture of two modulation spectra. (The samples tend to move as well.) For semiconductors one cannot use the electrolyte method in the vacuum ultraviolet for lack of a transparent electrolyte. The reverse-biased Schottky barrier method, utilizing a thin semitransparent metal layer on the surface, can be used for electroreflectance (ER) measurements on those semiconductors on which suitable barriers can be made. The modulating fields must be uniform over the penetration depth of the radiation in order to obtain simple lineshapes. This puts some restriction on the materials that can be studied this way, but it leads to very high resolution spectra in many cases, for the low-field spectra are third derivative, not first, spectra of the dielectric function [16]. For high resistivity semiconductors, the field can be applied in the plane of the surface between two stripe electrodes, but the fields obtainable are usually smaller than for the Schottky barrier method.

2.4 MAGNeTiC Field MOdUlation. - [21] usually leads to very small signals, which, in opaque samples, are very difficult to detect. Considerable amplification of the applied modulating field occurs in ferromagnetic materials, and the internal magnetization is modulated, leading to detectable signals. This is the magneto-optic Kerr effect, to date applied to several ferromagnetic metals in the vacuum ultraviolet.

\subsection{WAVELENGTH MODULATION SPECTROSCOPY. -} [22] and other methods to be reported below are not strictly modulation spectroscopies in that the samples themselves are not subjected to perturbations. In wavelength modulation spectroscopy, one installs an oscillating mirror or other device in the monochromator so that the emerging wavelength is $\lambda+\Delta \lambda \cos \omega t$. Then conventional lock-in techniques can give $\mathrm{d} R / \mathrm{d} \lambda$, if the wavelength dependence of the incident spectrum, the transmission of the optical system, and the detector sensitivity can be divided out. The increase in resolution of the derivative spectrum can be useful by itself, and by measuring spectra under various static conditions, e.g., at different temperatures, modulation spectra, e.g., thermoreflection spectra, can be simulated.

2.6 ENERGY DERIVATIVE SPECTROSCOPY. - [23] involves taking numerically the first and second derivatives of the measured spectra. This can be done almost on-line if the data are fed into a small on-site computer. In order to obtain a good derivative spectrum the signal-to-noise ratio of the original spectrum must be very high - far higher than needed for utilization of just the original spectrum. Again, spectra can be taken under various static conditions.

2.7 Composition modulation. - [24] involves two samples, e.g., one pure and one doped or alloyed. The beam strikes them alternately and the ratio of the difference in the two reflected or transmitted beams to their average is recorded as $\Delta R / R$ or $\Delta T / T$. This represents the derivative of $R$ or $T$ with respect to composition. In a variation of this method, the transmission of an alloy film is compared with composition-weighted thicknesses of its unalloyed constituents.

We now describe some of the results of modulation spectroscopy in the vacuum ultraviolet. Several recent reviews have appeared [25-27]. We will not cover energy derivative spectroscopy, only for lack of space, rather than lack of merit. There were a few measurements made before the advent of synchrotron radiation, although above about $6 \mathrm{eV}$, conventional continuum sources become relatively weak and good signal-to-noise ratios take a long time to obtain. Synchrotron radiation from synchrotrons is difficult to use for modulation spectroscopy, for each injection contains a different number of electrons, leading to a noisy amplitude of the monochromated beam on the sample. This has been removed successfully by proper beam monitoring and division of the reflected or transmitted signal by the monitor signal. Electron storage rings are almost ideal sources for modulation spectroscopy above $6 \mathrm{eV}$. The basic limitations to modulation spectroscopy as the photon energy increases are in the samples themselves. At high energies the reflectance falls so much that the reflected beam is too weak for measurements on a reasonable time scale. Modulated transmission holds some promise, however, and preliminary measurements have been encouraging. The other limitation is that at high energies the electron or hole lifetime often becomes short, broadening the spectrum to the point that derivative spectra are too small and broad to separate from the background. At high enough energies this limitation obtains for all materials, but for some selected transitions, the spectra at high energy are unexpectedly sharp.

3. Results. - 3.1 Metals. - The first VUV modulation spectroscopy was Scouler's thermomodulation study of $\mathrm{Au}$ to $10 \mathrm{eV}$ [28], followed by similar spectra on $\mathrm{Ni}$ [29]. The measurements on $\mathrm{Au}$ were later extended to $30 \mathrm{eV}$ using synchrotron radiation [30]. Figure 2 shows the measured $\Delta R / R$ spectrum and the differential dielectric function derived from it. Several of the structures have been assigned tentatively to critical points, but a definitive assignment is not yet at hand, for we are dealing with states many $\mathrm{eV}$ above the Fermi level, where band structure calculations often have convergence problems. Notable in figure 2 are the three structures just above $18 \mathrm{eV}$. These were originally interpreted [30] as transitions from the crystal-field and spin-orbit split d-bands to a flat band composed of $p$ - and f-like states. Subsequent ultraviolet photoemission measurements resolved only two peaks [31], and the differential dielectric function [26] indeed shows only 


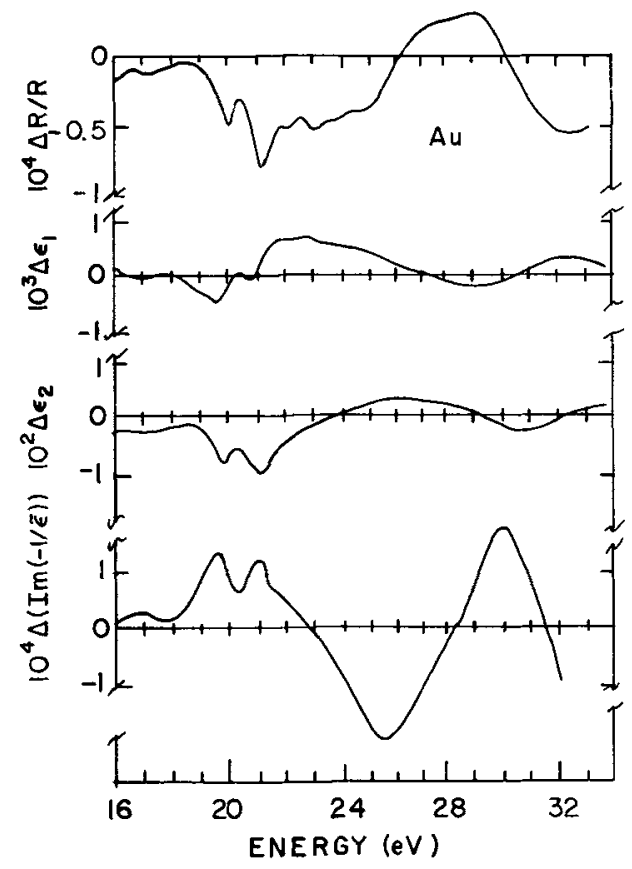

Frg. 2. - Thermoreflectance spectrum of Au and the differential dielectric function and differential loss function derived from it by Kramers-Kronig analysis. (From Refs. [26, 30].)

two peaks. (However, see later discussion.) The interpretation of these peaks is still tentative. Recent band calculations [32] show that there are a number of possible transitions for each, although a few can be ruled out by line shape considerations [26]. The calculation of electric dipole matrix elements should assist considerably in the interpretation. The observed peaks are surprisingly sharp, with a width of about $100 \mathrm{meV}$. They occur at the onset of d-to-p and d-to-f transitions, the majority of whose oscillator strength is delayed several $\mathrm{eV}$, giving rise to the reflectance peak which makes $\mathrm{Au}$ an effective coating for gratings in the vacuum ultraviolet. Such reflectance peaks occur in other fcc $5 \mathrm{~d}$ and $4 \mathrm{~d}$ metals, and, to a small extent in $\mathrm{Cu}$, but not in $3 \mathrm{~d}$ transition metals. All of these peaks show some changes in slope near their onsets, at least for those metals measured. Thermomodulation measurements on $\mathrm{Ag}$ and $\mathrm{Pt}$ show structure comparable to that of $\mathrm{Au}$, but not as sharp [33]. Evidently, as the d-band rises toward the Fermi level, the lifetime of the holes in it is reduced. The complete analysis of the $\Delta R / R$ spectra to give $\Delta \varepsilon$ is in progress [33].

Corresponding transitions occur in bcc and hcp transition metals but at lower energies. Thermoreflection measurements were carried out on Mo [34], but structures as numerous and sharp as those for $\mathrm{Au}$ in the corresponding region were not found. The early measurements were made on thin films, but for $\mathrm{Pt}$, better-resolved structures were found on annealed foil specimens.

These $\Delta \widetilde{\varepsilon}$ structures arise from d-electrons excited to final states which are probably fairly well localized, at least in Au. Additional measurements carried out by composition modulation on $\mathrm{Au}-\mathrm{Cu}$ alloys confirm this for the Au-rich end of the composition range [35]. In these measurements, the spectra of alloys were essentially weighted sums of the spectra of the constituents, implying localized transitions because the initial states, the d-bands, are known to form a common d-band in these alloys.

Recently Rosei et al. [36] have demonstrated that thermotransmission measurements on unsupported films of $\mathrm{Au}$ gave spectra in the visible that were better resolved than those taken by thermoreflectance on films on quartz. This work was soon extended to the vacuum ultraviolet, where well-resolved thermotransmission spectra of $\mathrm{Au}$ and $\mathrm{Ag}$ were obtained to $30 \mathrm{eV}$ [33]. This method has several advantages. The mass of the sample is very small, resulting in small time constants. Modulation frequencies of $100 \mathrm{~Hz}$ are possible and very large signals result, more than 10 times larger than in thermoreflection on similar films that are supported. The observed spectrum for $\mathrm{Au}$ in the $18-30 \mathrm{eV}$ region is close to that of $-\Delta \varepsilon_{2}$, but three sharp peaks are resolved just above $18 \mathrm{eV}$. Neglecting multiple reflections

$$
T=\frac{(1-R)}{(1+R)} \mathrm{e}^{-\alpha d} .
$$

(This leads to an optimum signal-to-noise ratio for a film thickness $d=2 / \alpha$, at normal incidence.) Then

$$
\begin{aligned}
\frac{\Delta T}{T} & =-d \Delta \alpha-\frac{2 R}{1-R^{2}} \frac{\Delta R}{R} \\
& =-\frac{2 \pi d}{n \lambda_{0}} \Delta \varepsilon_{2}-\frac{2 R}{1-R^{2}} \frac{\Delta R}{R} .
\end{aligned}
$$

That the observed spectra are nearly identical to the $-\Delta \varepsilon_{2}$ spectra obtained from Kramers-Kronig analysis on other samples means the second term, not measurable on these samples, is nearly negligible for these samples and the measured spectra may be interpreted directly. Moreover, $\Delta T / T$ spectra on $\mathrm{Au}$ films of different thicknesses give identical spectra, showing the dominance of the first term in equation (7). (Caution is necessary before neglecting $\Delta R / R$ with other materials or other wavelength regions.)

Kunz et al. [37-39] have carried out composition modulation measurements on ordered and disordered alloys of $\mathrm{Al}$ with several transition metals and on $\mathrm{Cu}-\mathrm{Ni}$, all in the soft $\mathrm{X}$-ray region. The measurements on $\mathrm{Cu}-\mathrm{Ni}$ [37] showed clearly that the $\mathrm{M}_{2,3}$ absorption of each constituent is localized on a single site. The Al $\mathrm{L}_{2,3}$ (an example is in figure 3) and transition metal $\mathbf{M}_{2,3}$ edges shifted upon alloying, and there were changes in the region several $\mathrm{eV}$ above the edges. The interpretation of the shifts could not be made on a single model, although density of 


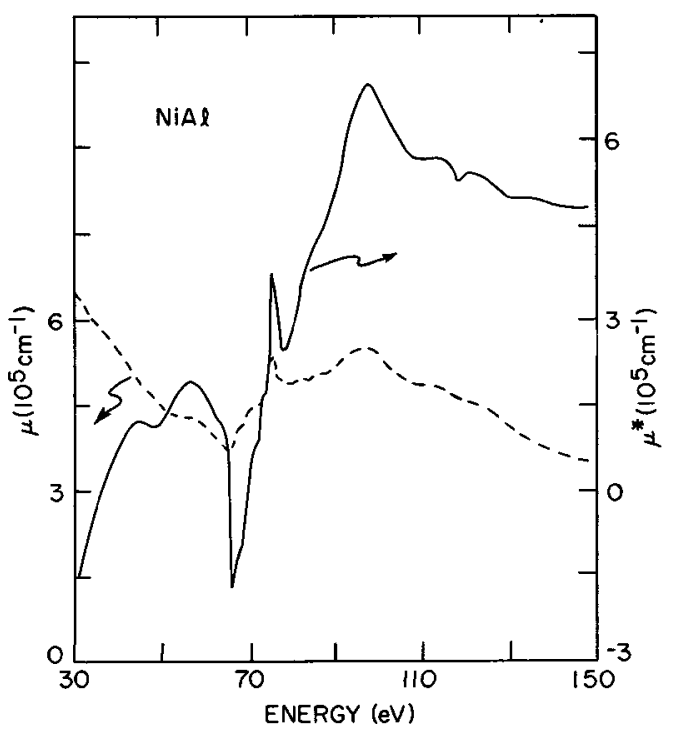

FIG. 3. - Absorption coefficient $\mu$ (solid) and a differential absorption coefficient $\mu^{*}$ (dashed) for NiAl. The latter is the difference between the absorption coefficient for $\mathrm{NiAl}$ and that of a film of $\mathrm{Ni}$ with the same number density of $\mathrm{Ni}$ atoms per unit area as in the alloy film. $\mu^{*}$ represents the absorption of the $\mathrm{Al}$ in the alloy if the $\mathrm{Ni}$ absorption is unchanged upon alloying, so that the $\mathrm{Al} \mathrm{L}_{2,3}$ edge in the alloy may be compared with that of Al. (From Ref. [39].)

states effects could account for the region above the edges in the ordered alloys.

Erskine has carried out magneto-optic Kerr effect measurements on ferromagnetic Gd [40] and $\mathrm{Ni}$ [41]. For a cubic metal the dielectric function is a diagonal tensor with one independent component. (Gd films may be considered cubic for our purposes.) The application of a magnetic field introduces off-diagonal components linear in the magnetization M. For simple geometries there is only one such component whose real part is proportional to the difference in absorption of left- and right-hand polarized radiation. Figure 4 shows such a spectrum for Gd

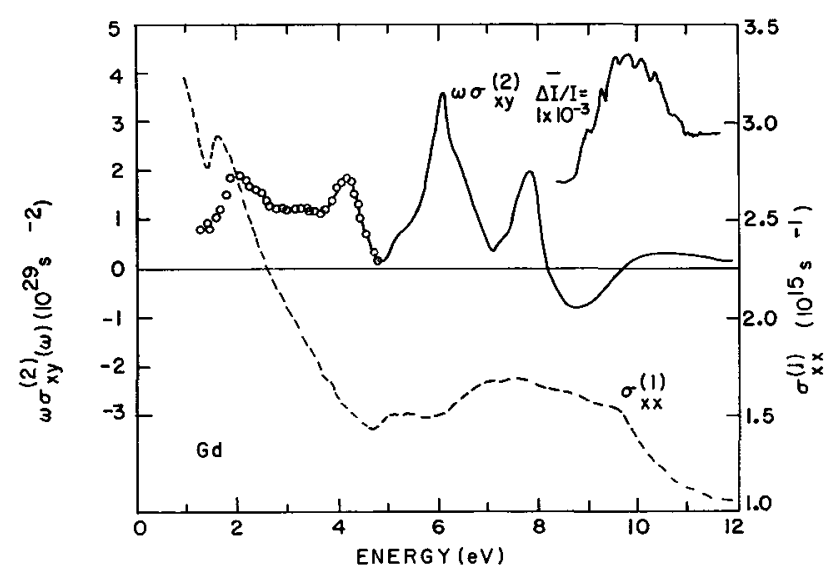

FIG. 4. - Conductivity (dashed) and absorptive component of the off-diagonal component of the magneto-conductivity tensor (solid) for $\mathrm{Gd}$. The inset shows additional fine structure in the directly measured spectrum (solid), attributed to multiplet structure. (From Ref. [40].) except that here the conductivity is displayed and the aforementioned absorption difference is described by the imaginary part of the off-diagonal conductivity tensor.

The microscopic calculation of this component is not physically transparent [42]. The transitions giving large magneto-optic absorption are not those contributing strongly to the conductivity; they are from states with large spin-polarization and spin-orbit splitting. In ferromagnetics, these usually are the dand f-electrons. The peak in $\operatorname{Im} \sigma_{x y}$ in figure 4 was attributed to the onset of transitions from the filled uf states to empty d-like conduction band states. This structure is more manifest in the magnetooptic spectrum than in the accompanying spectrum of the optical conductivity, $\operatorname{Re} \sigma_{x x}$.

3.2 SEmiconductors. - The first VUV modulation spectra on semiconductors were thermoreflection measurements to $9 \mathrm{eV}$ on several group IV and III-V semiconductors [43-45]. These were compared and similar transitions identified, making use of the Phillips-Van Vechten spectroscopic model for bands of partly ionic semiconductors. These spectra should resemble wavelength derivative spectra, and where they overlap with wavelength derivative spectra made below $6 \mathrm{eV}[46,47]$, they do, except for more small structures being resolved in the latter.

Schottky barrier electroreflectance measurements on $\mathrm{GaP}, \mathrm{GaAs}$ and $\mathrm{GaSb}$ in the $5-30 \mathrm{eV}$ region gave spectra rich in sharp structure [48-50]. Below about $20 \mathrm{eV}$ these structures arise from transitions between the valence band and conduction band. Many could be identified from calculated bands as arising from critical points, but others remain unidentified. Repeating these measurements under uniaxial stress could assist in the assignment.

At about $20 \mathrm{eV}$ the $\mathrm{Ga} 3 \mathrm{~d}$ electrons can be excited to the conduction bands. The case of $\mathrm{GaP}$ is the simplest, for the transitions are to the $\mathrm{X}_{6}$ conduction band minimum at threshold and do not overlap transitions to higher conduction band minima. The $\Delta R / R$ spectra were Kramers-Kronig analyzed to give $\Delta \varepsilon$ [51]. See figure 5. The $\Delta \varepsilon_{1}$ spectrum was decomposed into two spectra, identical in shape, but shifted and weighted by the expected effect of the spin-orbit interaction. This also gave the best least structure fit. The shape of one resulting component could be fit well (Fig. 5) by a theory of electroreflectance via the Franz-Keldysh effect at an excitonenhanced $\mathbf{M}_{0}$ critical point with broadening [52-54]. Estimates of the electric dipole matrix elements for transitions to $\Gamma, \mathrm{X}$ and $\mathrm{L}$, along with knowledge of the line shape for the $\mathrm{X}$ transitions in GaP, were used to interpret the corresponding spectra in $\mathrm{GaAs}$ and $\mathrm{GaSb}$. In $\mathrm{GaAs}$, the transitions to $\Gamma$ are weak, but detectable. The transitions to $\mathrm{L}$ occur below those to $X$ [55], meaning that the previous ordering of the $\mathrm{X}$ and $\mathrm{L}$ conduction band critical points had to be 

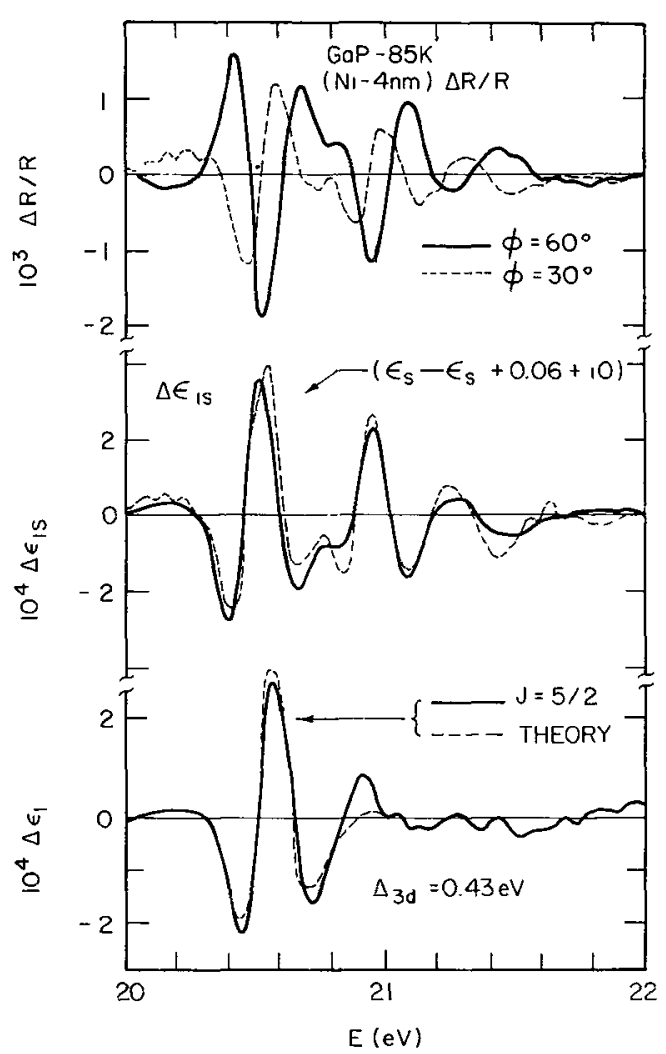

FIG. 5. - Electroreflectance spectrum of GaP obtained at two angles of incidence (top). Each was Kramers-Kronig analyzed to give the $\Delta \varepsilon_{2}$ spectrum shown in the center. Finally, this was decomposed into two spin-orbit components, one of which was fitted to theory (bottom). (From Ref. [51].)

incorrect [56]. (Subsequent resonant Raman scattering measurements [57] confirm this.)

The line shape fit of the $\Delta \varepsilon_{1}$ spectrum for GaP gave a value of $160 \mathrm{meV}$ for the core exciton binding energy and $160 \mathrm{meV}$ for the broadening parameter. A comparison of XPS data with the electroreflectance data gave $\mathrm{Ga} 3 \mathrm{~d}$ core exciton binding energies of 170,90 , and $90 \mathrm{meV}$ for $\mathrm{GaP}, \mathrm{GaAs}$, and GaSb [58], respectively, considerably larger than the effective mass energies of 38,16 , and $16 \mathrm{meV}$. (Larger values of the core exciton binding energies have been obtained using other criteria [59].)

The GaP spectra show no crystallographic anisotropy [51] which usually arises for valence band to conduction band ER spectra. The temperature dependence of the structures in $\mathrm{GaP}$ has also been measured and can be explained as arising solely from the temperature dependence of the final states. High resolution reflectance measurements recently yielded, when differentiated, structures corresponding to those in figure 3 [61].

Thermomodulation and electromodulation measurements were made on the $L_{2.3}$ edge of $\mathrm{Si}$ [62]. The latter yielded no spectrum, which result was interpreted as arising from a core exciton binding energy of at least $300 \mathrm{meV}$, although a value of $40 \mathrm{meV}$ had been used to fit the shape of the absorption edge [63]. The former yielded spectra which could be interpreted. It proved easier to measure energy derivative spectra at several temperatures rather than direct thermoreflection spectra. The spectra arose from the temperature dependence of the final states only.

It is clear that modulation spectroscopy of core transitions can be used to learn a great deal about the conduction bands, for the core levels exhibit no dispersion. The problem is to find a technique that works for a given core level. The Ga $3 \mathrm{~d}$ excitations do not occur strongly to final states localized on the group V atom. Attempts to measure ER spectra on GaAs in the region of the As 3d excitations failed because of low sample reflectivity at about $38 \mathrm{eV}$. A transmission method would be more appropriate.

3.3 Insulators. - The first VUV modulation spectra obtained out on insulators were by electrotransmission on several alkali iodides, carried out to $8.2 \mathrm{eV}$ by Menes $[64,65]$. The samples were thin films between semitransparent electrode films, and the field was longitudinal. Large signals due to the excitons were obtained. This was followed by thermoreflectance measurements on $\mathrm{MnO}, \mathrm{CoO}$ and $\mathrm{NiO}$ to $8 \mathrm{eV}$ [66]. The TR spectra revealed more structure than the reflectance spectra, except for MnO. They were used to assign temperature dependences to the structures found in the reflectance spectra.

These were followed by thermoreflectance measurements on a number of alkali halides to $9 \mathrm{eV}[67,68]$. The spectra were collected into groups of structures with similar line shapes and temperature coefficients, for the spectra were measured at two temperatures, and these groups of structures were assigned to excitons at $\Gamma, \mathrm{X}$ and $\mathrm{L}$.

Wavelength modulation spectra on the $R b 4 p$ core excitations in Rb-halides were taken at two temperatures using synchrotron radiation [64]. In the range $15-19 \mathrm{eV}$ there are more than 10 sharp structures. These were interpreted with a crystal field model of cation core excitons. The high resolution obtained made a study of the temperature dependence of these transitions easier to carry out.

Thermoreflection spectra were made to $30 \mathrm{eV}$ on $\mathrm{LiF}, \mathrm{KCl}$ and several $\mathrm{Rb}$-halides [70-72]. The reflectance spectrum of LiF is quite simple, but interpretation has not been easy. Moreover, at about $25 \mathrm{eV}$ there is a peak in the electron energy loss function and the imaginary part of the dielectric function which has been given a variety of interpretations, including the simultaneous excitation of two valence band excitons. The valence band exciton gives a $\Delta \varepsilon_{2}$ spectrum primarily due to the shift of the band edge with temperature (see Fig. 6). The $\Delta \varepsilon_{2}$ structure at about $22.2 \mathrm{eV}$, roughly twice the valence exciton energy, has a completely different shape, arising from the temperature dependence of the broadening, so it 


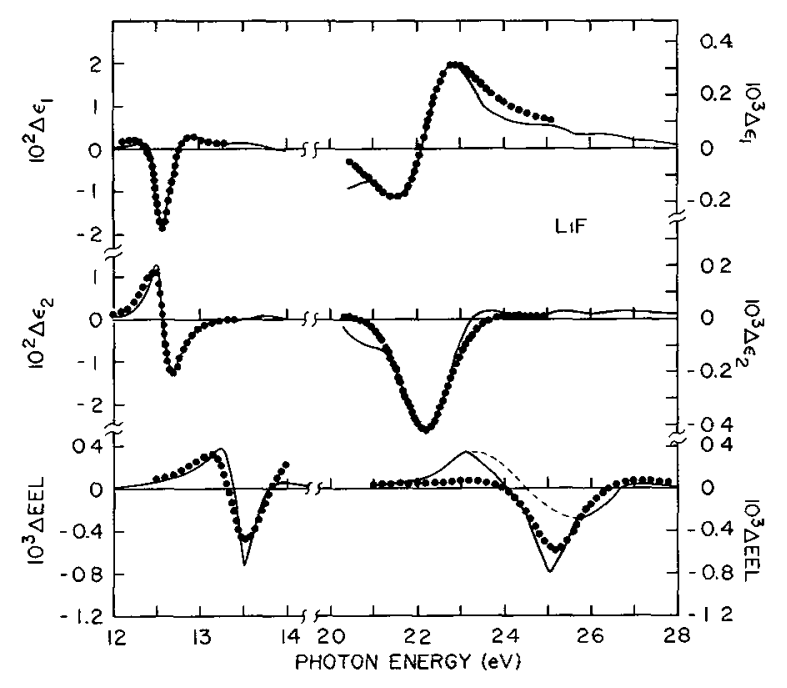

FIG. 6. - Differential dielectric function and differential loss function for Lif derived from thermoreflectance spectra (solid) The dots represent the spectra of a single Lorentzian oscillator shifted (lower-energy structure) or broadened (higher-energy structure) by the temperature modulation. The dashed line is the remaining contribution to the differential loss function. (From Ref. [70].)

seems not to be due to two valence band excitons. The corresponding structure in the differential electron energy loss function, $\Delta \operatorname{Im}(-1 / \widetilde{\varepsilon})$, is more complicated, but it can be decomposed into two structures. One, like the structure in $\Delta \varepsilon_{2}$, is broadened by temperature. It is the longitudinal counterpart of the peak in $\Delta \varepsilon_{2}$ and represents a longitudinal exciton or group of interband transitions. The other, overlapping, structure has the appearance of a valence band plasmon, shifted in energy by thermal expansion. This overlapping of a plasmon and a longitudinal interband excitation or exciton appears in other alkali halides as well, both longitudinal excitations falling at a gap or minimum in the interband transverse interband excitation spectrum [70].

Acknowledgments. - Much of the work described above was carried out in collaboration with Dr. C. G. Olson, Dr. D. E. Aspnes, Dr. M. Piacentini, Dr. J. H. Weaver, and Dr. R. Rosei, whose contributions are gratefully acknowledged. Much of the work was carried out at the Synchrotron Radiation Center of the University of Wisconsin, supported by NSF contract No. DMR-74-15089. This work was supported by the U.S. Energy Research and Development Administration, Division of Physical Research, and the manuscript written at the Aspen Center for Physics.

\section{References}

[1] Cardona, M., Modulation Spectroscopy (Academic Press, New York) 1967.

[2] Seraphin, B. O. and Bottka, N., Phys. Rev. 145 (1966) 628.

[3] Aspnes, D. E., J. Opt. Soc. Am. 63 (1973) 1380.

[4] Aspnes, D. E., Olson, C. G. and LynCh, D. W., J. Appl. Phys. 47 (1976) 602.

[5] Balzarotti, A., Colavita, E., Gentile, S. and Rosei, R., Appl. Opt. 14 (1975) 2412.

[6] Barz, B., Semiconductors and Semimetals, Willardson. R. K. and Beer, A. C , eds., Vol. 9 (Academıc Press. New York) 1972, p. 316.

[7] Rosei, R. and Lynch, D. W., Phys. Rev. B 5 (1972) 3883.

[8] BALSLEV, I., Semiconductors and Semimetals, Willardson, R. K. and Beer, A. C., eds., Vol. 9 (Academic Press, New York) 1972, p. 403

[9] Pollak, F. H., Surf. Sci. 37 (1973) 863.

[10] Sell, D. D., Surf. Sci. 37 (1973) 896.

[11] Gerhardt, V., Phys. Rev. 172 (1968) 651.

[12] Szczepanek, P. and Glosser, R., Solid State Commun. 15 (1974) 1425.

[13] Seraphin, B. O., Semiconductors and Semumetals, Willardson, R. K. and Beer, A. C., eds., Vol. 9 (Academic Press, New York) 1972, p. 1.

[14] Blossey, D. F. and Handere, P., Semiconductors and Semimetals, Willardson, R. K. and Beer, A. C., eds., Vol. 9 (Academic Press, New York) 1972, p. 257.

[15] Aspnes, D. E. and BotTKA, N., Semiconductors and Semimetals, Willardson, R. K. and Beer, A. C., eds., Vol. 9 (Academic Press, New York) 1972, p. 457.

[16] Aspnes, D. E. and Rowe, J. E., Phys. Rev. B 5 (1972) 4022.

[17] Fisher, J E. and Aspnes, D E., Phys. Status Solidi $(h) 55$ (1973) 9.

[18] AsPnes, D. E., Surf. Sci. 37 (1973) 418.

[19] Nishino, T. and Hamakawa, Y., Optical Properties of Solids New Developments, Seraphin, B. O., ed. (North-Holland. Amsterdam) 1976, p. 255.
[20] Reun, V., Surf. Sci. 37 (1973) 443.

[21] AgGarwal, R. C., Semiconductors and Semimetals, Willardson, R. K. and Beer, A. C., eds., Vol. 9 (Academic Press, New York) 1972, p. 151.

[22] SHEN, Y. R., Surf. Sci. 37 (1973) 522, and references therem.

[23] Sell, D. D., Appl. Opt. 9 (1970) 1926.

[24] Gudat, W., Kunz, C. and Karlau, J., Appl. Opt. 13 (1974) 1412.

[25] Aspnes, D. E., Nuovo Cimento 39B (1977) 337.

[26] Piacentini, M., Nuovo Cimento 39B (1977) 682.

[27] AsPNES, D. E., Festkorperprobleme XVHI : Advances in Solid State Phvsics, Treusch. J., ed. (Pergamon-Vieweg, Braunschwe1g) 1977, p. 235.

[28] Scouler, W. J., Phys. Rev. Lett. 18 (1967) 445.

[29] Hanus, J., Feinleib, J. and Scouler, W. J., Phys. Rew. Lett. $19(1967) 16$.

[30] Olson, C. G., Piacenimin, 'M. and Linch, D. W., Phys. Rev. Lett. 33 (19/4) 644.

[31] Hermanson, J., Anderson, J. and Lapeyre, G. J., Phys. Rev. B 12 (1975) 5410.

[32] Christensen, N. E., Phys. Rev. B 13 (1976) 2698.

[33] Olson, C. G., Rosei, R. and LynCH, D. W., to be published.

[34] Weaver, J. H., Olson, C. G., Lynch, D. W. and Piacentini, M., Solid State Commun. 16 (1975) 163.

[35] Beaglehole, D. and Thiéblemont, B., Nuovo Cimento 39B (1977) 477.

[36] Meuti, M., Coluzza, C., Quaresima, C. and Rosei, R., Appl. Opt. 1 (1977) 217.

[37] Gudar, W. and Kunz. C. Phys. Status Solidi (b) 52 (1972) 433.

[38] Hagemann, H.-J., Gudat, W. and Kunz, C., Solid State Commun. 15 (1974) 655.

[39] Hagemann, H.-J., Gudat, W. and Kunz, C., Phvs. Status Solidi (b) 74 (1976) 507.

[40] Erskine, J. L., Phys. Rev. Lett. 37 (1976) 157.

[41] Erskine, J. L., Physica B 89B (1977) 83. 
[42] Erskine, J. L. and Stern, E. A., Phys, Rev. B 12 (1975) 5016.

[43] Nosenzo, L., Reguzzoni, E. and Samoggia, G., in Proc. of Intl. Conf. on the Physics of Semiconductors, Warsaw, 1972 (Polish Scientific Publishers, Warsaw) 1972, p. 1409.

[44] Guizzetti, G., Nosenzo, L., Reguzzoni, E. and Samoggia, G., Surf. Sci. 37 (1973) 508.

[45] Guizzetri, G., Nosenzo, L., Reguzzoni, E. and Samoggia, G., Phys. Rev. B 9 (1974) 640.

[46] Walter, J. P., ZucCa, R. R. L., Cohen, M. L. and Shen, Y. R., Phys. Rev. Lett. 24 (1970) 102.

[47] ZuCCA, R. R. L. and Shen, X. R., Phys. Rev. B 1 (1970) 2668.

[48] Aspnes, D. E. and Olson, C. G., Phys. Rev. Lett. 33 (1974) 1605.

[49] Aspnes, D. E., Olson, C. G. and LynCh, D. W., Phys. Rev. B 12 (1975) 2527.

[50] Aspnes, D. E., Olson, C. G. and Lynch, D. W., Phys. Rev. B 15 (1976) 5331

[51] Aspnes, D. E., Olson, C. G. and Lynch, D. W., Phys. Rev. B 14 (1976) 2534.

[52] Blossey, D. F., Phys. Rev. B 3 (1971) 1382.

[53] Weinstein, F. C., Dow, J. D. and Lao, B. Y., Phys. Rev. B 4 (1971) 3502.

[54] Blossey, D. F., Phys. Rev. B 2 (1970) 2976.

[55] Aspnes, D. E., Olson, C. G. and LynCh, D. W., Phys. Rev. Lett. 37 (1976) 766.

[56] Aspnes, D. E., Phys. Rev. B 14 (1976) 5331.

[57] Trommer, R. and Cardona, M., Solid State Commun. 21 (1977) 153 . [s]] Aspnes, D. E., Olson, C. G and Lynch. D. W., Proc 13th Int. Conf. on the Physics of Semiconductors, Rome, Fumi, F. G., ed., 1976 (Tipografia Marves, Rome, 1977) p. 1000 .

[59] Thiry, P., Petroff, Y., Pinchaux, R., Chelikowsky, J. and CoHen, M. L., Solid State Commun. 70 (1976) 1107.

[60] Aspnes, D. E., Olson, C. G. and Lynch, D. W., Phys. Rev. Lett: 36 (1976) 1563.

[61] Aspnes, D. E., Saile, V., Sprussel, G. and Cardona, M., to be published.

[62] Bauer, R. S., Bachrach, R. Z., Aspnes, D. E. and MCMenaMIN, J. C., Nuovo Cimento 39B (1977) 409.

[63] Altarelli, M. and Dexter, D. L., Phys. Rev. Lett. 29 (1972) 110.

[64] Stevens, M. and Menes, M., Phys. Lett. A 27 (1968) 472.

[65] Menes, M., Phys. Rev. B 10 (1974) 4469.

[66] Messick, L., Walker, W. C. and Glosser, R., Phys. Rev. B 6 (1972) 3941.

[67] Nosenzo, L., Reguzzoni, E. and Samoggia, G., Phys. Rev. Lett. 28 (1972) 1388.

[68] Guizetti, G., Nosenzo, L. and Reguzzoni, E., Phys. Rev. B 15 (1977) 5921.

[69] Zierau, W. and Skibowski, M., J. Phys. C 8 (1975) 1671.

[70] Lynch, D. W., Piacentini, M. and Olson, C. G., Phys. Rev. Lett. 35 (1975) 1658.

[71] Piacentini, M., Lynch, D. W. and Olson, C. G., Phys. Rev. B 13 (1976) 5530.

[72] Piacentini, M., Olson, C. G. and Lynch, D. W., to be published. 